\footnotetext{
${ }^{1}$ Национальный медицинский исследовательский центр эндокринологии, Москва, Российская Федерация

${ }^{2}$ Сибирский государственный медицинский университет, Томск, Российская Федерация

${ }^{3}$ Тюменский государственный медицинский университет, Тюмень, Российская Федерация

${ }^{4}$ Морозовская детская городская клиническая больница, Москва, Российская Федерация

${ }_{5}^{5}$ Областная детская клиническая больница имени Н.В. Дмитриевой, Рязань, Российская Федерация
}

ОБОСНОВАНИЕ. Статистика последних лет свидетельствует о значительном росте заболеваемости и распространенности сахарного диабета в детской и подростковой популяции. По данным исследований, целевых показателей гликированного гемоглобина ( $\left.\mathrm{Hb}_{1 c}\right)$ достигают лишь 38\% пациентов. Повысить эффективность медицинской помощи детям с сахарным диабетом 1-го типа возможно с помощью современных технологий, в том числе непрерывного мониторирования глюкозы.

ЦЕЛЬ - оценить эффективность амбулаторного наблюдения детей и подростков с сахарным диабетом 1-го типа при регулярном использовании профессионального непрерывного мониторинга глюкозы.

МЕтОДЫ. В исследование были включены дети и подростки, отвечающие следующим критериям: возраст $\geq 8$ и <18 лет; сахарный диабет 1-го типа с длительностью заболевания не менее 1 года; инсулинотерапия путем множественных инъекций инсулина; неадекватный гликемический контроль сахарного диабета 1-го типа: содержание $\mathrm{HbA}_{1 c}$ 7,5\% и выше и/или дети и подростки с частыми эпизодами гипогликемии (чаще 4 раз/нед, зафиксированные глюкометром) или с эпизодами тяжелой гипогликемии в анамнезе; подписанное информированное согласие. Всем пациентам исходно и через 12 нед после включения в исследование проводилось исследование уровня НbА ${ }_{1 c^{\prime}}$ а также осуществлялось непрерывное мониторирование глюкозы в течение 6 дней. По результатам непрерывного мониторирования глюкозы проводилась регистрация показателей гликемии и суточных доз инсулина; давались оценка и коррекция проводимого лечения, рекомендации по самоконтролю и наблюдению. Мониторирование глюкозы проводилось в течение не менее 120 и не более 144 ч с использованием непрерывного мониторирования глюкозы «слепым» методом iPro2 (Medtronic, США).

РЕЗУЛЬТАТЫ. Всего во всех центрах в исследование было включено 99 детей в возрасте 8-18 лет. Средний уровень $\mathrm{HbA}_{1 c^{\prime}}$ исходно составлявший 9,6\%, статистически значимо снизился к концу исследования до 8,9\%. Таким образом, снижение уровня $\mathrm{HbA}_{1 с}$ к концу исследования составило 0,72\%, при этом доля пациентов, которые достигли целевого уровня $\mathrm{HbA}_{1 c}$ (определяемого как <7,5\%), была статистически значимо выше к концу исследования (15,5 и 2\% соответственно; $\mathrm{p}<0,05)$. За время исследования у пациентов отмечалась тенденция к снижению показателей среднего уровня и вариабельности гликемии к концу исследования, однако статистическая значимость была достигнута только в отношении среднего уровня гликемии ( $\mathrm{p}=0,04)$. Проводимая инсулинотерапия, определяемая по среднесуточным дозам инсулина длительного и короткого действия, статистически значимо не изменилась к концу исследования. За время исследования частота эпизодов диабетического кетоацидоза и тяжелой гипогликемии статистически значимо не отличалась от исходного уровня.

ЗАКЛючЕНИЕ. У детей с неудовлетворительным гликемическим контролем сахарного диабета 1-го типа использование профессионального непрерывного мониторирования глюкозы является безопасным и эффективным в отношении гликемического контроля методом.

КЛЮЧЕВЫЕ СЛОВА: сахарный диабет 1-го типа у детей, сахарный диабет 1-го типа у детей и подростков, система непрерывного мониторирования глюкозы.

\title{
THE EFFICACY OF OUTPATIENT MONITORING OF CHILDREN AND ADOLESCENTS WITH TYPE 1 DIABETES WITH REGULAR USE OF PROFESSIONAL CONTINUOUS GLUCOSE MONITORING
}

(c) Valentina A. Peterkova', Dmitry N. Laptev', Andrey O. Emelyanov*, Yulia G. Samoylova², Elena B. Khramova³ Elena E. Petriaikina ${ }^{4}$, Irina G. Rybkina ${ }^{4}$, Alla Yu. Filimonova ${ }^{5}$ 
${ }^{1}$ National Medical Research Center of Endocrinology, Moscow, Russian Federation

${ }^{2}$ Siberian State Medical University, Tomsk, Russian Federation

${ }^{3}$ Tumenian State Medical University, Tyumen, Russian Federation

${ }^{4}$ Morozov Children City Clinical Hospital, Moscow, Russian Federation

${ }^{5}$ Regional Children Clinical Hospital named after N.V. Dmitrieva, Ryazan, Russian Federation

BACKGROUND: According to research, only $38 \%$ of patients reach glycated hemoglobin targets. It is possible to improve the effectiveness of medical care for children with T1D using modern technologies, including continuous glucose monitoring (CGM).

AIMS: To evaluate the effectiveness of outpatient monitoring of children and adolescents with T1D with regular use of professional continuous glucose monitoring.

METHODS: The inclusion criteria: age 8-12 years; T1D at least 1 year; insulin therapy by multiple injections of insulin; inadequate glycemic control of T1D: $\mathrm{HbA}_{1 \mathrm{c}}$ level of $7.5 \%$ and higher and / or children and adolescents with frequent episodes of hypoglycemia (usually 4 times a week) or with a history of severe hypoglycemia; signed informed consent. All patients initially and 12 weeks after inclusion in the study conducted a study of the level of $\mathrm{HbA}_{1 c^{\prime}}$ and also performed CGM for 6 days. Based on the results of CGM, glycemia indicators and daily doses of insulin were recorded, treatment was evaluated and corrected, and recommendations for self-monitoring were made. Glucose monitoring was carried 120-144 hours using the blind method iPro2 (Medtronic, USA).

RESULTS: In all, 99 children aged 8-18 years were included in the study in all centers. The decrease in the level of $\mathrm{HbA}_{1 \mathrm{c}}$ by the end of the study was $0.72 \%$, while the proportion of patients who reached the target level of $\mathrm{HbA}_{1 \mathrm{c}}$ (defined as $<7.5 \%$ ) was statistically significantly higher at the end of the study $(15.5 \%$ and $2 \%$, respectively; $p<0.05)$. During the study, patients showed a trend towards a decrease in the average level and variability of glycemia by the end of the study, however, statistical significance was achieved only in relation to the average level of glycemia $(p=0.04)$. Conducted insulin therapy, determined by the average daily doses of long-acting and short-acting insulin, did not statistically significantly change at the end of the study. The frequency of DKA episodes and severe hypoglycemia did not statistically significantly differ from the initial level.

CONCLUSIONS: For children with poor glycemic control of T1D, the use of professional CGM is effective in terms of glycemic control and a safe method.

KEYWORDS: diabetes mellitus type 1, adolescent, child, blood glucose, glycemic, metabolic, glucose monitoring.

\section{ОБОСНОВАНИЕ}

Статистика последних лет свидетельствует о значительном росте заболеваемости и распространенности сахарного диабета в детской и подростковой популяциях [1]. В России, по данным Федерального регистра сахарного диабета, в настоящее время насчитывается более 33 тыс. детей и подростков с сахарным диабетом 1-го типа. При этом в последние годы отмечается тенденция к увеличению числа пациентов с дебютом заболевания в детском и подростковом возрасте.

В 2014 г. по инициативе ФГБУ «Эндокринологический научный центр» Минздрава России (в настоящее время ФГБУ «Национальный медицинский центр эндокринологии» Минздрава России) и Фонда поддержки и развития филантропии «КАФ» при финансовой поддержке консорциума «Альфа-групп» проведено исследование оценки качества оказания эндокринологической помощи детям и подросткам в 6 регионах Российской Федерации. Поскольку показатели гликированного гемоглобина (гликогемоглобин, $\mathrm{HbA}_{1 c}$ ) являются в настоящее время наиболее значимыми критериями оценки контроля компенсации сахарного диабета у детей, многие параметры медицинской помощи были проанализированы в контексте этого показателя. В результате исследования установлено, что определение $\mathrm{HbA}_{1 c}$ доступно во всех регионах большинству пациентов, однако, по данным амбулаторных карт, регулярно его измеряют 20\% детей, по данным опроса родителей, - 57\%. Согласно полученным данным, целевых показателей $\mathrm{HbA}_{1 с}$ достигают лишь 38\% пациентов [2].

При анализе данных была выявлена зависимость уровня $\mathrm{HbA}_{1 c}$ от следующих важнейших факторов: ежегодного посещения детского эндокринолога с частотой 1 раз в 3 мес (не больше, но и не меньше); ежедневного измерения уровня глюкозы с частотой 4 раза в день и более; комплекса социальных факторов риска (воспитание ребенка только матерью, бедность, отсутствие работы). Факторами риска ухудшения компенсации являлись подростковый возраст и удаленное проживание от медицинских организаций, в которых оказывалась специализированная медицинская помощь [2].

Не выявлено значимой связи уровня $\mathrm{HbA}_{1 c}$ с разнообразными медицинскими факторами, включая наличие инсулиновой помпы, при том что доля детей, получающих инсулинотерапию в помповом режиме, составляла в различных регионах от 21 до 47\%. Себестоимость установки и использования инсулиновых помп очень высокая, поэтому необходимо повысить эффективность использования за за счет совершенствования медицинского наблюдения $[2,3]$.

Повысить эффективность медицинской помощи детям с сахарным диабетом 1-го типа возможно с помощью 
современных технологий, в том числе непрерывного мониторирования глюкозы.

Использование непрерывного мониторирования глюкозы описано в стандартах лечения детей с сахарным диабетом 1-го типа, однако в практическом здравоохранении данная методика используется нечасто [2].

Трудности достижения компенсации и неспособность врачей детских эндокринологов амбулаторного и стационарного звена постоянно контролировать состояние больного сформировали необходимость организации амбулаторных центров мониторинга для проведения консультаций с квалифицированными специалистами по различным вопросам достижения высокой компенсации углеводного обмена и повышения роли больного в проведении самоконтроля и лечении заболевания.

Непрерывное профессиональное мониторирование глюкозы крови - наиболее объективный метод оценки гликемического контроля у больных сахарным диабетом. Данное исследование не требует специальной подготовки пациента и обычно проводится в течение до 6 сут. Система непрерывного мониторирования глюкозы и данные, полученные с ее помощью, позволяют не только выявить определенные тенденции суточных колебаний гликемии с регистрацией всех эпизодов гипо- и гипергликемий, но и выяснить причины их возникновения, выявить скрытые гипогликемии, а затем эффективно скорректировать инсулинотерапию с учетом образа жизни пациента.

Использование системы непрерывного мониторирования глюкозы, по данным многочисленных зарубежных и отечественных авторов [4-11], позволяет улучшить показатели гликемии у пациентов с сахарным диабетом.

\section{ЦЕЛЬ}

Оценить эффективность амбулаторного наблюдения детей и подростков с сахарным диабетом 1-го типа при регулярном использовании профессионального непрерывного мониторинга глюкозы.

\section{МЕТОДЫ}

\section{Дизайн исследования}

Проведено 12-недельное проспективное открытое неконтролируемое клиническое исследование.

\section{Критерии соответствия}

Критерии включения: дети и подростки в возрасте $\geq 8$ и <18 лет; наличие диагноза сахарного диабета 1-го типа с длительностью заболевания не менее 1 года; использование инсулинотерапии путем множественных инъекций инсулина; неадекватный гликемический контроль сахарного диабета 1-го типа ( $\left.\mathrm{HbA}_{1 с} \geq 7,5 \%\right)$ и/или частые (>4 раз/нед) эпизоды гипогликемии, зафиксированные глюкометром, или эпизоды тяжелой гипогликемии в анамнезе; подписанное информированное согласие.

Критерии исключения: беременность; хирургическое вмешательство в последние 4 нед перед включением в исследование; активные заболевания печени, включая гепатиты (аспартатаминотрансфераза, аланинаминотрансфераза >2,5 раза выше верхней границы нормы); активная нефропатия - нефротический синдром или гломерулонефрит (скорость клубочковой фильтрации $<60$ мл/мин/1,73 м²); ВИЧ-инфекция по данным анамнеза; злокачественные новообразования (включая заболевания крови).

\section{Условия проведения}

Проект реализован на базе 5 клинических центров Российской Федерации:

ФГБУ «Национальный медицинский исследовательский центр эндокринологии» Минздрава России;

ФГБОУ ВО «Сибирский государственный медицинский университет» Минздрава России;

ГБОУ ВПО «Тюменский государственный медицинский университет» Минздрава России;

ГБУЗ «Морозовская детская городская клиническая больница Департамента здравоохранения города Москвы»;

ГБУ РО «Областная детская клиническая больница имени Н.В. Дмитриевой».

\section{Описание медицинского вмешательства}

Общее количество очных визитов для каждого пациента - 4, с интервалом 12 нед между визитами 1 и 3 и 6 дней между визитами 1-2 и 3-4.

Непрерывное мониторирование глюкозы. Мониторирование глюкозы проводилось в течение не менее 120 и не более 144 ч с использованием профессиональной системы непрерывного мониторирования глюкозы «слепым» методом iPro2 (Medtronic, США). Сенсор устанавливается подкожно и измеряет уровень глюкозы в интерстициальной жидкости. Поскольку концентрация глюкозы в интерстициальной жидкости и капиллярной крови может быть разной, после считывания данных проводится калибровка системы с использованием показаний глюкометра.

Система непрерывного мониторирования глюкозы проводит измерение показателей каждые 5 мин, границы измерения - от 2,2 до 22,2 ммоль/л. При использовании системы «слепым» методом пользователь не имеет доступа к текущим показателям глюкозы, а по окончании исследования результаты работы сенсора предоставляются лечащему врачу для ретроспективного анализа.

Все пациенты были предварительно обучены пользованию системой непрерывного мониторирования глюкозы - калибровке и уходу за прибором. Для калибровки использовались данные об уровне гликемии, зафиксированные в дневниках пациентов. Также при калибровке фиксировалась информация о проводимой инсулинотерапии с указанием вида, дозы и времени введения инсулина.

Самоконтроль глюкозы. Всем пациентам были предоставлены глюкометр Contour TS (Bayer, Швейцария) и тест-полоски к нему на все время исследования с рекомендацией проведения самоконтроля не менее 4 раз/сут и фиксацией времени измерения и показателей в дневнике самоконтроля.

Регистрация показателей. Регистрация и анализ показателей гликемии (средний уровень гликемии, вариабельность гликемии (SD), гликемический 8-точечный профиль) проводились с использованием программного обеспечения CareLink iPro2 (Medtronic MiniMed, США) за предшествующий дню визита шестидневный период. 
Таблица 1. Исходная характеристика пациентов с сахарным диабетом 1-го типа

\begin{tabular}{lccc}
\hline \multicolumn{1}{c}{ Пациенты, $\boldsymbol{n = 9 9}$} & M (SD) & Минимум & Максимум \\
\hline Возраст, лет & $12,5(2,6)$ & 8,0 & 17,8 \\
Женщины / Мужчины & $53 / 46$ & - & - \\
Длительность заболевания, лет & $5,3(3,3)$ & 1,0 & 15,2 \\
HbА $_{1 c^{\prime}} \%$ & $9,6(1,6)$ & 7,0 & 14,0 \\
\hline
\end{tabular}

Примечание. Данные представлены в виде M (SD), минимального и максимального значений. НbА

Данное программное обеспечение позволяет сформировать отчеты, содержащие данные и графическую информацию об основных показателях гликемического контроля: показатели гликемии в течение суток, средняя гликемия, стандартное отклонение среднего уровня гликемии, частота показателей в пределах выше и ниже целевого диапазона и др.

Регистрация показателей среднесуточных доз инсулина проводились пациентами в индивидуальных дневниках самоконтроля.

\section{Исходы исследования}

Основной исход исследования. Изменение концентрации $\mathrm{HbA}_{1 с}$ к концу исследования по сравнению с исходным уровнем и доля пациентов, достигших значения $\mathrm{HbA}_{1 с}$ менее 7,5\% к концу исследования.

Дополнительные исходы исследования. Изменение к концу исследования по сравнению с исходным уровнем следующих показателей: средний уровень гликемии, вариабельность гликемии (SD), гликемический 8-точечный профиль; частота эпизодов диабетического кетоацидоза, тяжелой гипогликемии за время исследования.

\section{Методы регистрации исходов}

Всем пациентам исходно и через 12 нед после включения в исследование проводились стандартный осмотр и антропометрия, определение концентрации $\mathrm{HbA}_{1 c}$ методом жидкостной хроматографии на анализаторе DS5 Glycomat (DrewScientific, Нидерланды), или методом реакции агглютинации моноклональных антител на анализаторе DCA Vantage Analyzer (Siemens, Германия), или ферментативным методом на анализаторе BS-400 (Mindray, Китай); также осуществлялось непрерывное мониторирование глюкозы в течение 6 дней, по результатам которого проведены регистрация показателей гликемии и суточных доз инсулина, оценка и коррекция проводимого лечения; даны рекомендации по самоконтролю и наблюдению.

\section{Статистический анализ}

Статистическая обработка полученных результатов была произведена с использованием статистического пакета STATISTICA (StatSoft, США). При нормальном распределении количественного признака данные представлены в виде среднего значения и его стандартного отклонения - M (SD) или в виде среднего значения и его стандартной ошибки - M \pm SEM. Различие между количественными признаками оценивалось с помощью метода Манна-Уитни, между качественными признаками - с помощью двустороннего точного критерия Фишера, между частотой острых осложнений (диабетический кетоацидоз, тяжелая гипогликемия) - с помощью двухстороннего Z-критерия. Взаимосвязь между двумя показателями оценивалась с использованием корреляционного анализа методом Спирмена. Для всех статистических методов значение $p<0,05$ считалось статистически значимым.

\section{РЕЗУЛЬТАТЫ}

Объекты (участники) исследования

Всего в исследование было включено 99 детей в возрасте от 8 до 18 лет. Исходная характеристика пациентов представлена в табл. 1.

Основные результаты исследования

Гликированный гемоглобин. Средняя концентрация $\mathrm{HbA}_{1 c^{\prime}}$ исходно составлявшая 9,6\%, статистически значимо снизилась к концу исследования до 8,9\% (табл. 2, рис. 1).

Таким образом, снижение концентрации $\mathrm{HbA}_{1 с}$ к концу исследования относительно исходного составило 0,72\%, при этом доля пациентов, которые достигли целевого показателя концентрации $\mathrm{HbA}_{1 c}$ (определяемого как $<7,5 \%)$, была статистически значимо выше к концу исследования по сравнению с исходным уровнем (15,5 и 2\% соответственно; $p<0,05 ;$ рис. 2).

Показатели гликемии (средняя гликемия, вариабельность гликемии). За время исследования у пациентов отмечалась тенденция к снижению показателей среднего уровня и вариабельности гликемии к концу исследования, однако статистическая значимость была достигнута только в отношении среднего уровня гликемии (см. табл. 2, рис. 3; $p=0,04$ ).

Гликемический профиль. Несмотря на то что к концу исследования отмечалась тенденция к более низким показателям гликемии по сравнению с исходным уровнем, все показатели гликемического профиля статистически значимо не изменились (см. табл. 2, рис. 4).

Инсулинотерапия. Проводимая инсулинотерапия, определяемая по среднесуточным дозам инсулина длительного и короткого действия, статистически значимо не изменилась к концу исследования по сравнению с исходным уровнем (см. табл. 2, рис. 5).

Острые осложнения. За время исследования частота эпизодов диабетического кетоацидоза и тяжелой гипогликемии статистически значимо не отличалась от исходного уровня (см. табл. 2, рис. 6). 
Таблица 2. Показатели гликемического контроля, гликемического профиля, инсулинотерапии, острых осложнений

\begin{tabular}{|c|c|c|c|c|}
\hline Показатель & Исходно & 12 нед & Дельта & $p$ \\
\hline \multicolumn{5}{|c|}{ Гликемический контроль } \\
\hline $\mathrm{HbA}_{1 \mathrm{c}^{\prime}} \%$ & $9,6(1,6)$ & $8,9(1,6)$ & $-0,7(1,2)$ & 0,002 \\
\hline Средний уровень гликемии, ммоль/л & $10,4(2,2)$ & $9,8(2,1)$ & $-0,6(2,1)$ & 0,04 \\
\hline Вариабельность гликемии SD, ммоль/л & $4,7(2,7)$ & $4,5(2,9)$ & $-0,3(1,6)$ & $\mathrm{H} / 3$ \\
\hline \multicolumn{5}{|c|}{ Гликемический профиль 8 точек } \\
\hline Гликемия при пробуждении, ммоль/л & $9,5(2,3)$ & $9,2(2,4)$ & $-0,2(2,5)$ & $\mathrm{H} / 3$ \\
\hline Гликемия перед завтраком, ммоль/л & $9,9(2,6)$ & $9,4(2,6)$ & $-0,5(3)$ & $\mathrm{H} / 3$ \\
\hline Гликемия через 120 мин после завтрака, ммоль/л & $11,5(3,4)$ & $10,7(3,2)$ & $-0,8(3,6)$ & $\mathrm{H} / 3$ \\
\hline Гликемия перед обедом, ммоль/л & $10,5(3,3)$ & $9,8(2,8)$ & $-0,7(3,5)$ & $H / 3$ \\
\hline Гликемия через 120 мин после обеда, ммоль/л & $10,6(3)$ & $9,8(3,3)$ & $-0,7(3,9)$ & $\mathrm{H} / 3$ \\
\hline Гликемия перед ужином, ммоль/л & $9,9(3,1)$ & $9,6(2,8)$ & $-0,4(3,3)$ & $H / 3$ \\
\hline Гликемия через 120 мин после ужина, ммоль/л & $9,7(2,7)$ & $9,7(3,1)$ & $0,1(3,4)$ & $H / 3$ \\
\hline Гликемия перед сном, ммоль/л & $10(2,6)$ & $9,6(2,7)$ & $-0,4(2,8)$ & $\mathrm{H} / 3$ \\
\hline \multicolumn{5}{|c|}{ Инсулинотерапия } \\
\hline Среднесуточная доза инсулина, ЕД & $39(17,6)$ & $41,8(17,9)$ & $2,9(5,6)$ & $\mathrm{H} / 3$ \\
\hline Среднесуточная доза инсулина длительного действия, ЕД & $18,4(9,5)$ & $19,6(10,1)$ & $1,2(2,3)$ & $\mathrm{H} / 3$ \\
\hline Среднесуточная доза инсулина короткого действия, ЕД & $20,7(9,8)$ & $21,9(10)$ & $1,1(3,5)$ & $H / 3$ \\
\hline \multicolumn{5}{|c|}{ Острые осложнения } \\
\hline Число эпизодов тяжелой гипогликемии, кол-во/12 нед & $0(0,3)$ & $0(0)$ & - & $\mathrm{H} / 3$ \\
\hline Число эпизодов кетоацидоза, кол-во/12 нед & $0(0,1)$ & $0(0,1)$ & - & $H / 3$ \\
\hline
\end{tabular}

Примечание. Данные представлены в виде M (SD). $\mathrm{HbA}_{1 c}$ — гликированный гемоглобин; н/з — статистически незначимые различия.

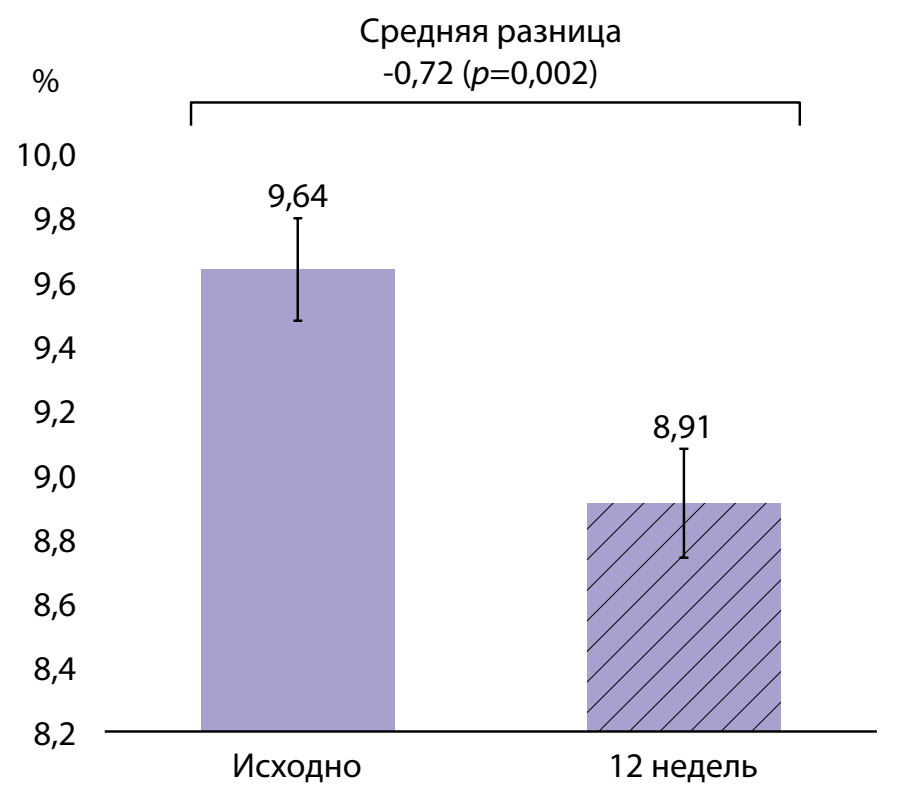

Рис. 1. Динамика уровня гликированного гемоглобина $\left(\mathrm{HbA}_{1 c}\right)$ за время исследования в группах тяжелой гипогликемии и диабетического кетоацидоза.

Примечание. Данные представлены в виде $\mathrm{M} \pm \mathrm{SEM}$.

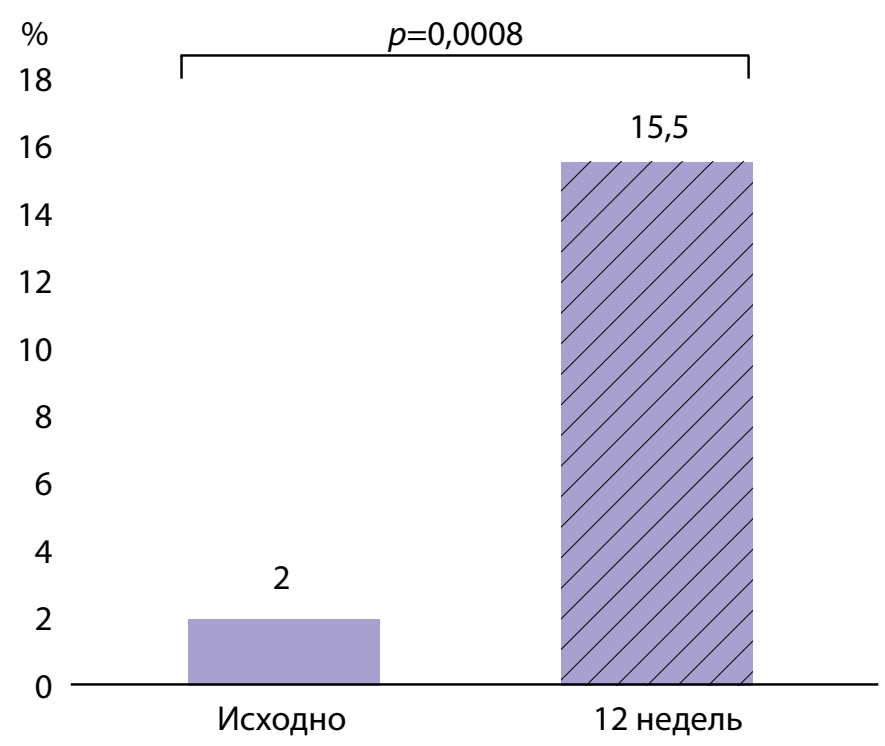

Рис. 2. Динамика компенсации углеводного обмена. Примечание. Данные представлены в виде доли (\%) пациентов с уровнем гликированного гемоглобина $<7,5 \%$. 


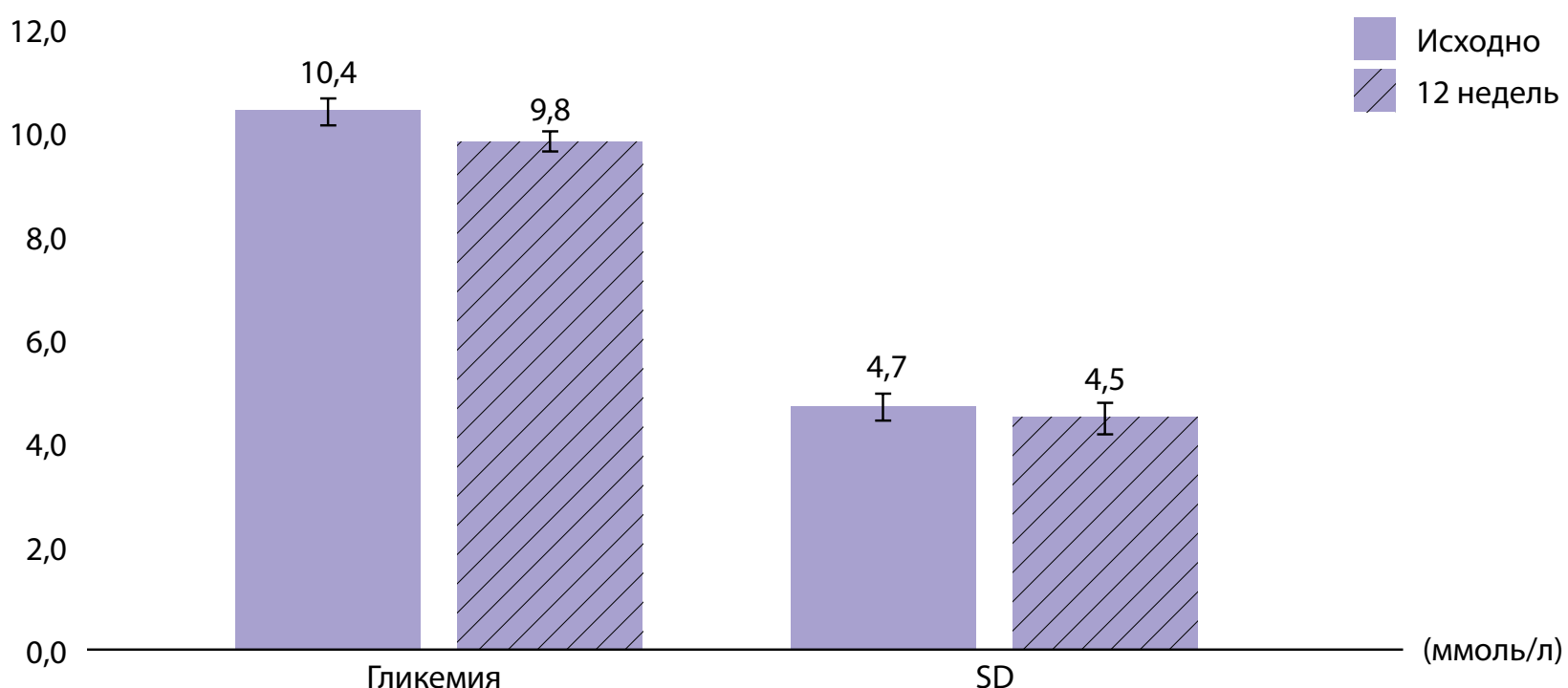

Рис. 3. Показатели гликемического контроля исходно и к концу исследования.

Примечание. Данные представлены в виде $\mathrm{M} \pm \mathrm{SEM}$. Для всех показателей уровень $p>0,05$ между визитами.

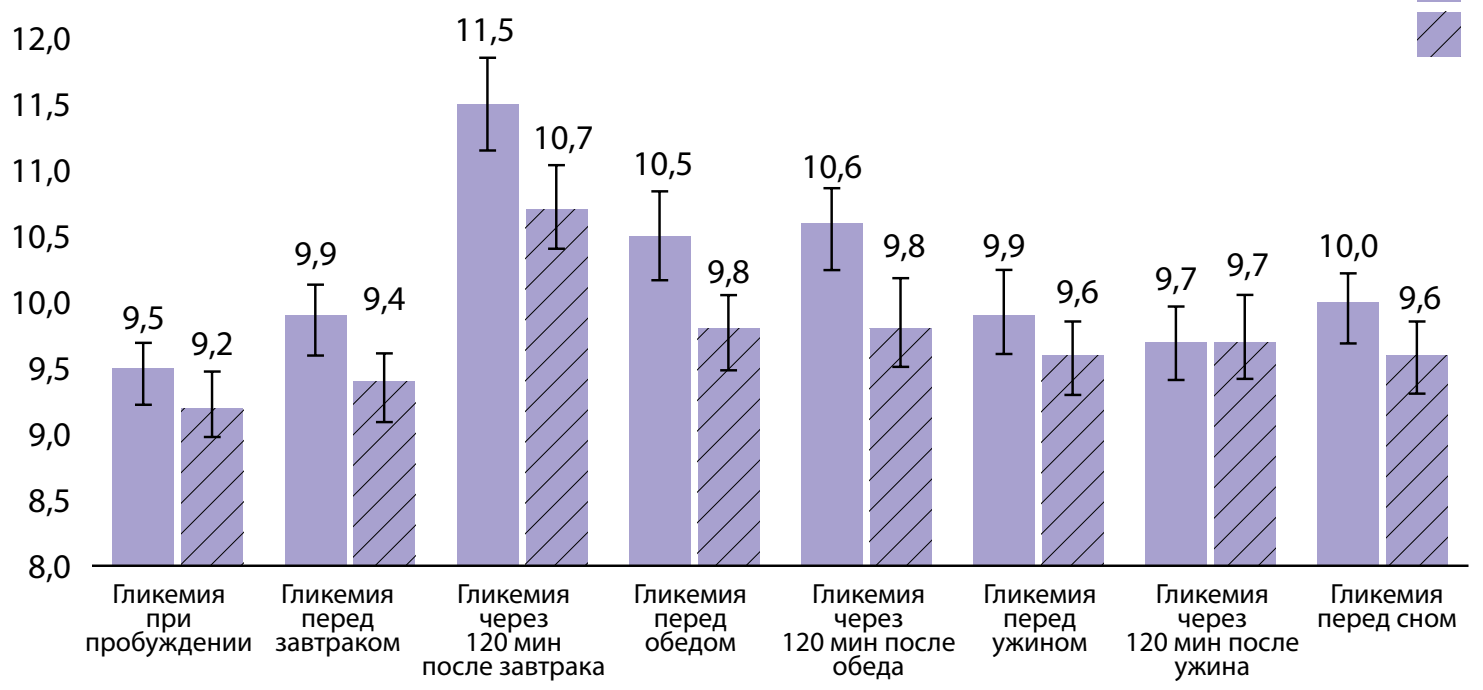

Исходно

12 недель

Рис. 4. Показатели гликемического профиля (8 точек) исходно и к концу исследования.

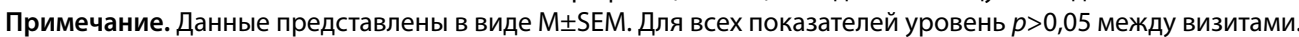

48,0

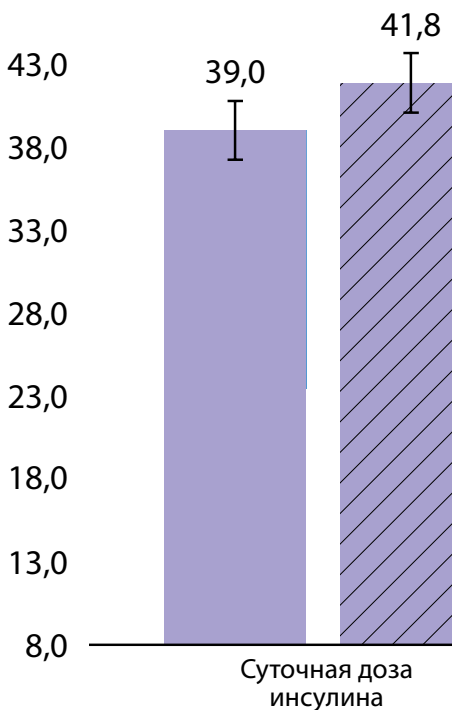

\section{1,8}
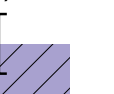


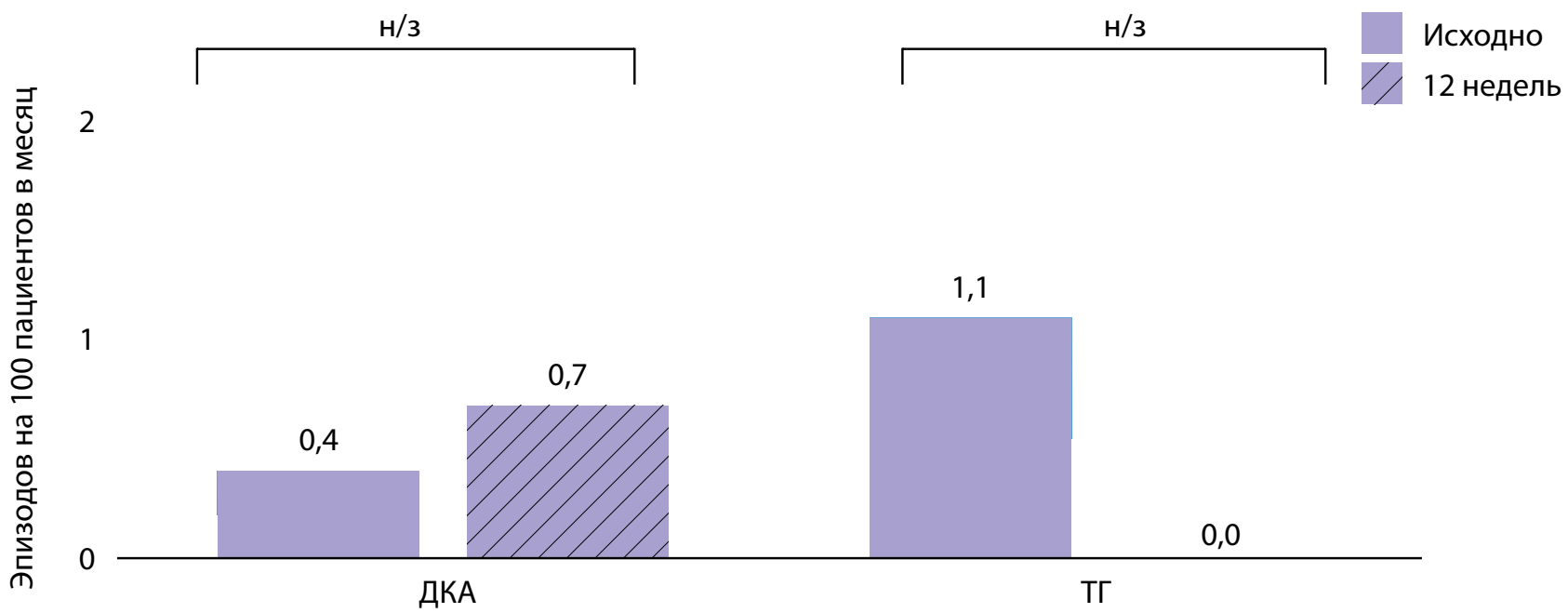

Рис. 6. Частота эпизодов тяжелой гипогликемии и диабетического кетоацидоза исходно и за время исследования.

Примечание. Данные представлены в виде числа случаев на 100 пациентов в месяц. ТГ — тяжелая гипогликемия; ДКА — диабетический кетоацидоз.

\section{ОБСУЖДЕНИЕ}

В нашей работе за 12 нед наблюдения было показано, что использование профессионального непрерывного мониторирования глюкозы при оказании медицинской помощи детям с сахарным диабетом 1-го типа в амбулаторных условиях статистически значимо улучшает показатели гликемического контроля, определяемые по содержанию $\mathrm{HbA}_{1 c}$ и среднего уровня гликемии. Снижение концентрации $\mathrm{HbA}_{1 c}$ сопровождается статистически значимым увеличением числа пациентов с компенсированным гликемическим контролем (уровень $\mathrm{HbA}_{1 с}$ менее 7,5\%).

Гликированный гемоглобин является одним из важнейших показателей эффективности лечения у пациентов с сахарным диабетом 1-го типа, т.к. непосредственно связан с риском поздних осложнений сахарного диабета 1-го типа [12]. Полученные результаты по эффективности непрерывного мониторирования глюкозы во многом согласуются с данными проведенных к настоящему времени зарубежных и отечественных исследований, однако большинство этих работ касаются преимуществ использования непрерывного мониторирования глюкозы в реальном времени, т.е. собственно непрерывного контроля глюкозы и коррекции лечения по его результатам [7, 10, 11-13]. Также большинство авторов указывают на ограниченную эффективность кратковременного мониторирования в связи с небольшой длительностью наблюдения и отсутствием возможности видеть показатели гликемии в реальном времени, в связи с чем профессиональному непрерывному мониторированию глюкозы уделяется меньше внимания в клинической практике, а основная роль отводится использованию в клинических исследованиях. При этом с экономической точки зрения стоимость непрерывного мониторирования глюкозы в реальном времени при постоянном его использовании более чем в 12 раз выше в сравнении с профессиональным непрерывным мониторированием глюкозы в течение 6 дней за 3 мес. Наше исследование указывает на потенциальные возможности использования профессионального крат- ковременного непрерывного мониторирования глюкозы при амбулаторном наблюдении с целью повышения эффективности проводимого лечения у детей с неудовлетворительным гликемическим контролем сахарного диабета 1-го типа.

Более высокая эффективность проводимого лечения на основе профессионального непрерывного мониторирования глюкозы, вероятно, отражает возможности детских эндокринологов выявлять проблемные места в лечении, такие как скрытые эпизоды гипогликемии, эпизоды постпрандиальной гипергликемии и высокой вариабельности гликемии; оценивать адекватность схемы инсулинотерапии и дозировок инсулина, а также проводить наглядное обучение пациентов, полагаясь на данные мониторинга глюкозы.

Среднесуточная потребность в инсулине не различалась исходно и в конце исследования между группами. Это может указывать на отсутствие связи полученного эффекта с изменением суточных доз инсулина.

В нашей работе за время исследования частота эпизодов острых осложнений сахарного диабета 1-го типа, таких как тяжелая гипогликемия и диабетический кетоацидоз, статистически значимо не отличалась от исходного уровня, что указывает на безопасность указанного подхода по сравнению с традиционным амбулаторным наблюдением.

\section{ЗАКЛЮЧЕНИЕ}

У детей с неудовлетворительным гликемическим контролем сахарного диабета 1-го типа использование профессионального непрерывного мониторирования глюкозы является безопасным и эффективным в отношении гликемического контроля методом.

\section{ДОПОЛНИТЕЛЬНАЯ ИНФОРМАЦИЯ}

Источник финансирования. Работа выполнена в рамках национальной благотворительной программы помощи детям с эндокринными заболеваниями «Альфа-Эндо» при финансовой поддержке фонда «КАФ». Компания АО «Байер» предоставила для исследования 
глюкометры Contour TS и тест-полоски к ним.

This work was carried out as part of the Alfa-Endo national charitable program for helping children with endocrine diseases with financial support from the KAF Foundation. Bayer JSC provided Contour TS glucometers and test strips for them for research.

Конфликт интересов. Авторы декларируют отсутствие явных и потенциальных конфликтов интересов, связанных с публикацией настоящей статьи.
Участие авторов. Научное руководство, дизайн и планирование исследования - В.А. Петеркова; выгрузка данных из регистра, анализ и статистическая обработка полученных данных, написание текста Д.Н. Лаптев; ведение регистра помповой инсулинотерапии, выгрузка данных из регистра, написание и редактирование текста - А.О. Емельянов, ведение регистра помповой инсулинотерапии, выгрузка данных из регистра - Ю.Г. Самойлова, Е.Б. Храмова, Е.Е. Петряйкина, И.Г. Рыбкина, А.Ю. Филимонова.

\section{СПИСОК ЛИТЕРАТУРЫ | REFERENCES}

1. Dedov II. Shestakova MV, Peterkova VA, et al. Diabetes mellitus in children and adolescents according to the Federal diabetes registry in the Russian Federation: dynamics of major epidemiological characteristics for 2013-2016. Diabetes mellitus. 2018;20(6):392-402. doi: https://doi.org/10.14341/DM9460.

2. Петеркова В.А., Швабский О.Р., Карпушкина А.В., Витебская А.В. Отчет об основных результатах исследования потребностей пациентов и системы здравоохранения для улучшения качества медико-социальной помощи детям с эндокринными заболеваниями. - М., 2014. - 48 c. [Peterkova VA, Shvabskii OR, Karpushkina AV, Vitebskaia AV. Otchet ob osnovnykh rezul'tatakh issledovaniia potrebnostei patsientov i sistemy zdravookhraneniia dlia uluchsheniia kachestva mediko-sotsial'noi pomoshchi detiam s endokrinnymi zabolevaniiami. Moscow; 2014. 48 p. (In Russ).]

3. Дедов И.И., Петеркова В.А., Кураева Т.Л., Лаптев Д.Н. Инсулиновая помпа. Помощь врачу и пациенту для эффективного управления диабетом. - М., 2014. - 126 с. [Dedov II, Peterkova VA, Kuraeva TL, Laptev DN. Insulinovaia pompa. Pomoshch' vrachu i patsientu dlia effektivnogo upravleniia diabetom. Moscow; 2014. 126 p. (In Russ).]

4. Chase HP, Roberts MD, Wightman C, et al. Use of the GlucoWatch biographer in children with type 1 diabetes. Pediatrics. 2003;111(4 Pt 1):790-794. doi: https://doi.org/10.1542/peds.111.4.790.

5. Kaufman FR, Gibson LC, Halvorson M, et al. A pilot study of the continuous glucose monitoring system: clinical decisions and glycemic control after its use in pediatric type 1 diabetic subjects. Diabetes Care. 2001;24(12):2030-2034. doi: https://doi.org/10.2337/diacare.24.12.2030.

6. Ludvigsson J, Hanas R. Continuous subcutaneous glucose monitoring improved metabolic control in pediatric patients with type 1 diabetes: a controlled crossover study. Pediatrics. 2003;111(5 Pt 1):933-938. doi: https://doi.org/10.1542/peds.111.5.933.
7. Battelino T, Conget I, Olsen B, et al. The use and efficacy of continuous glucose monitoring in type 1 diabetes treated with insulin pump therapy: a randomised controlled trial. Diabetologia. 2012;55(12):3155-3162. doi: https://doi.org/10.1007/s00125-012-2708-9.

8. El-Laboudi AH, Godsland IF, Johnston DG, Oliver NS. Measures of glycemic variability in type 1 diabetes and the effect of realtime continuous glucose monitoring. Diabetes Technol Ther. 2016;18(12):806-812. doi: https://doi.org/10.1089/dia.2016.0146.

9. Pickup JC, Freeman SC, Sutton AJ. Glycaemic control in type 1 diabetes during real time continuous glucose monitoring compared with self monitoring of blood glucose: meta-analysis of randomised controlled trials using individual patient data. BMJ. 2011;343:d3805. doi: https://doi.org/10.1136/bmj.d3805.

10. Phillip M, Danne T, Shalitin S, et al. Use of continuous glucose monitoring in children and adolescents. Pediatr Diabetes. 2012;13(3):215-228. doi: https://doi.org/10.1111/j.1399-5448.2011.00849.x.

11. Лаптев Д.Н. Преимущество помповой инсулинотерапии и систем мониторирования гликемии в реальном времени в отношении снижения частоты эпизодов гипогликемии у детей и подростков с сахарным диабетом 1 типа // Проблемы эндокринологии. - 2014. - T.60. — №2. - C. 24-30. [Laptev DN The advantages of insulin pump therapy and real time glucose monitoring systems as the tools for reducing the frequency of hypoglycemic episodes in the children and adolescents with type 1 diabetes mellitus. Problems of endocrinology. 2014;60(2):24-30. (In Russ).] doi: https://doi.org/10.14341/probl201460224-30.

12. DCCT/EDIC Research Group; de Boer IH, Sun W, Cleary PA, et al. Intensive diabetes therapy and glomerular filtration rate in type 1 diabetes. N Engl J Med. 2011;365(25):2366-2376. doi: https://doi.org/10.1056/NEJMoa1111732.

13. Battelino T, Phillip M, Bratina N, et al. Effect of continuous glucose monitoring on hypoglycemia in type 1 diabetes. Diabetes Care. 2011;34(4):795-800. doi: https://doi.org/10.2337/dc10-1989.

\section{ИНФОРМАЦИЯ ОБ АВТОРАХ [AUTHORS INFO]}

*Емельянов Андрей Олегович, к.м.н., ведущий научный сотрудник [Andrey O. Emelyanov, MD, PhD]; aдpec: Россия, 117036, Москва, ул. Дм. Ульянова, д. 11 [address: 11 Dm. Ulyanov str., 117036 Moscow, Russia]; e-mail: endiab@mail.ru, SPIN-код: 8110-5540, ORCID: http://orcid.org/0000-0002-3742-0231

Петеркова Валентина Александровна, д.м.н., профессор, академик PAH [Valentina A. Peterkova, MD, PhD, Professor]; e-mail: peterkovava@hotmail.com, SPIN-код: 4009-2463, ORCID: http://orcid.org/0000-0002-5507-4627

Лаптев Дмитрий Никитич, д.м.н. [Dmitry N. Laptev, MD, PhD]; e-mail: laptevdn@ya.ru, SPIN-код: 2419-4019,

ORCID: http://orcid.org/0000-0002-4316-8546

Самойлова Юлия Геннадьевна, д.м.н., профессор [Yulia G. Samoylova, MD, PhD, Professor]; e-mail: samoilova_y@inbox.ru, SPIN-код: 8644-8043, ORCID: http://orcid.org/0000-0002-2667-4842

Храмова Елена Борисовна, д.м.н. [Elena B. Khramova, MD, PhD]; e-mail: doctor.khramova@gmail.com, SPIN-код: 2462-3440, ORCID: http://orcid.org/0000-0001-8968-3925

Петряйкина Елена Ефимовна, д.м.Н., професcop [Elena E. Petriaikina, MD, PhD, Professor]; e-mail: lepet_morozko@mail.ru, SPIN-код: 5997-7464, ORCID: http://orcid.org/0000-0002-8520-2378

Рыбкина Ирина Георгиевна, к.м.н. [Irina G. Rybkina, MD, PhD]; e-mail: ribkinairina@mail.ru

Филимонова Алла Юрьевна, доцент кафедры [Alla Yu. Filimonova, MD]; e-mail: alla.filimonova.65@mail.ru, ORCID: https://orcid.org/0000-0002-4795-670X/ 


\section{ЦИТИРОВАТЬ:}

Петеркова В.А., Лаптев Д.Н., Емельянов А.О., Самойлова Ю.Г., Храмова Е.Б., Петряйкина Е.Е., Рыбкина И.Г., Филимонова А.Ю. Оценка эффективности амбулаторного наблюдения детей и подростков с сахарным диабетом 1-го типа при регулярном использовании профессионального непрерывного мониторинга уровня глюкозы // Проблемы эндокринологии. — 2020. — Т.66. — №1. — С. 14-22. doi: https://doi.org/10.14341/probl12200

\section{TO CITE THIS ARTICLE:}

Peterkova VA, Laptev DN, Emelyanov AO, Samoylova YuG, Khramova EB, Petriaikina EE, Rybkina IG, Filimonova AYu. The Efficacy of Outpatient Monitoring of Children and Adolescents With Type 1 Diabetes With Regular Use of Professional Continuous Glucose Monitoring. Problems of Endocrinology. 2020;66(1):14-22. doi: https://doi.org/10.14341/probl12200 